\title{
¿Es posible la mejora educativa desde los centros? El papel de la organización escolar en los procesos internos de cambio
}

\author{
Noelia Ceballos López, Ángela Saiz Linares \\ Universidad de Cantabria (España)
}

\section{RESUMEN}

Analizamos el funcionamiento de algunas dimensiones de la organización escolar que han posibilitado que una experiencia innovadora inicialmente circunscrita a dos aulas de una escuela de Cantabria se haya ampliado progresivamente y convertido en un proyecto que implica a todo el centro educativo. Este estudio de los procesos internos de cambio en la organización escolar, llevado a cabo en el curso académico 2017/2018, se realiza a partir de un estudio etnográfico de este caso escogido por sus características diferenciales y particulares, buscando responder a una cuestión fundamental: ¿cuál es la relevancia de la organización escolar en los procesos de mejora? Las técnicas de recogida de información han sido: observación participante, grupos de discusión y análisis documental. Realizamos un análisis de contenido que revela algunas claves que facilitan la innovación: la revisión de las culturas escolares a partir de procesos dialógicos que implican a los diferentes agentes de la comunidad; la creación de condiciones estructurales (tiempos y espacios) destinados al intercambio; un equipo directivo que dinamiza la colaboración y genera dichas estructuras físicas desde formas de liderazgo distribuido; la recuperación de la autonomía pedagógica como clave fundamental para desarrollar cambios coherentes con la realidad educativa del centro.

Palabras clave: Organización y gestión, liderazgo, autonomía educativa, innovación pedagógica, etnografía

\section{Is it possible to improve education from the schools? The role of the school organisation in the internal processes of change}

\begin{abstract}
We analyze the functioning of some dimensions of the school organization that have enabled that an innovative experience initially circumscribed to two classrooms of a school in Cantabria has progressively expanded and become a project that involves the whole school. This study of the internal processes of change in school organization, carried out in 2017/2018, is based on an ethnographic study of this case chosen for its differential and particular characteristics, and is aimed at answering one fundamental question: what is the relevance of the school organization in the processes of educational improvement? Our sample refers to all agents of the school and the information gathering techniques are: participant observations, focus groups and document analysis. We carry out a content analysis that reveals some keys that facilitate innovation that involve the whole school: the revision of school cultures based on dialogical processes that involve the different agents of the community; the creation of structural conditions (times and spaces) destined for exchange; a leadership team that dynamizes the collaboration and generates these physical structures from distributed leadership; the recovery of pedagogical autonomy as a fundamental key to develop changes coherent with the educational reality of the school.
\end{abstract}

Keywords: Organization and management, Leadership, Educational autonomy, Educational innovations, Ethnography 


\section{Introducción}

La idea de que la educación se encuentra en crisis constituye un lugar común en las sucesivas reformas legislativas (Hopkins, 2013; San Fabián, 2011). A pesar de los continuos intentos reformistas, muchos estudios confirman su escasa incidencia en las políticas y prácticas de las escuelas (González, 2009; Pascual, Larraguibel, Zenteno y Guarda, 2016), destacando la desatención a la cultura de la organización escolar entre las principales causas (Álvarez-Arregui y Arreguit, 2019; Gather y Maulini, 2010; Gimeno, 2006; San Fabián, 2011).

Ante esta realidad, en este artículo analizamos algunas claves de la organización escolar que posibilitan los procesos internos de cambio. A través de un estudio etnográfico, desarrollado en el curso académico 2017-2018, analizamos el CEIP Manuel Llano (Cantabria), que hace unos años emprendió una innovación localizada en dos aulas que progresivamente fue tornando en un proyecto de centro que penetró hondamente en la cultura escolar. En nuestro análisis, trataremos de responder a algunos interrogantes: ¿cuál es la relevancia de la organización escolar en los procesos de mejora educativa? ¿Qué papel juega la cultura escolar en estos procesos? ¿Y el entramado de relaciones? ¿Y la estructura organizativa? ¿Y los procesos de planificación y desarrollo de los proyectos educativos? La organización escolar es el marco en el que se desarrollan los procesos de cambio educativos (González, 2009, Pérez-Gomar, 2016; San Fabián, 2011). Concebir la escuela como una unidad de mejora (Antúnez y Gairin, 2013; Cantón y Pino, 2014), implica asumir que los procesos de cambio son complejos y requieren tiempo para su asentamiento, pues dependen estrechamente de sus actores, del conocimiento que producen y de las condiciones organizativas que enmarcan la acción (Colás-Bravo, Jiménez y Rosado, 2014).

Por otro lado, los aspectos organizativos se configuran atendiendo a los aspectos funcionales (tareas y roles) y a los personales-relacionales (Fielding y Moss, 2011). En el momento actual dominado por las perspectivas neoliberales, se prioriza cada vez más una organización donde prima el poder individual sobre la responsabilidad colectiva, donde los aspectos funcionales devienen en un medio para legitimar los planteamientos de eficacia, rendimiento y competitividad. Nosotras, por el contrario, abogamos por aproximaciones democráticas que sitúan el diálogo en el centro de la vida escolar y los aspectos organizativos al servicio de las personas, los procesos de mejora y los proyectos compartidos (Fielding y Moss, 2011). La configuración de espacios basados en la responsabilidad compartida y el compromiso con el bien común nuclean dichos procesos de mejora (Slee, 2011; Thomson y Hall, 2015). A continuación, trataremos de definir las dimensiones más relevantes de la organización escolar desde esta perspectiva de comunidad democrática (Gather y Maulini, 2010; González, 2009; Kools y Stolls, 2016; Porter, Lawler y Hackman, 1975; Pérez-Gomar, 2016).

Una dimensión fundamental es la cultura escolar, referida al conjunto de teorías, ideas, rituales, hábitos y prácticas, construidas a lo largo del tiempo, que subyacen a lo que se piensa y hace en la organización (González, 2009). Algunas de ellas son declaradas formalmente mientras otras pertenecen al ámbito de lo implícito. Las escuelas, más que estructuras y roles organizados, constituyen grupos sociales cohesionados, a lo largo de la historia, en torno a una red de relaciones, valores y dinámicas sobre las que se configura la acción educativa (López-Yáñez, Sánchez-Moreno y Nicastro, 2002). Para promover mejoras se torna esencial indagar sobre estas culturas escolares que permitan movilizar ideas, creencias y modos de hacer.

Destacamos una segunda dimensión que informa sobre el entramado relacional y comunicacional y la toma de decisiones escolares (González, 2009). En este esqueleto social se definen las relaciones de poder y se dirime la capacidad de influir en la toma de decisiones, que aparece solo en parte determinada por los roles y funciones formales. Una escuela que quiere desarrollar un proyecto compartido está exhortada a entender el poder y cómo se negocia desde una posición no jerárquica, donde los distintitos agentes tienen la posibilidad de formar parte de los procesos de toma de decisiones (Fielding y Moss, 2011; Mitra, 2018).

Refiriendo a esta dimensión relacional es esencial abordar el papel del liderazgo escolar como clave en la construcción de un proyecto colectivo (Bolívar y Murillo, 2017; Leithwood, Harris, y Hopkins, 2008; Leiva, Gairin y Guerra (2019); Mulford, 2006; Pascual et al., 2016). Desde una perspectiva comunitaria, el liderazgo debe situar el aprendizaje en el centro de la vida escolar y poner al servicio de dicho proyecto las estructuras organizativas (Day, Gu y Sammons, 2016; Grootenboer, Edwards-Groves y Rönnerman, 2015; Kools y Stoll, 2016), abogando por planteamientos democráticos y participativos (Diamond y Spillane, 2016; Martínez-Valdivia, García-Martínez e Higueras-Rodríguez, 2018; Segal, Pollak y Lefstein, 2017). Esta consideración se enfrenta a la que promueve la LOMCE, que estimula los personalismos y concentra en la figura del director la competencia decisoria.

Finalmente, las investigaciones más recientes destacan cómo, a pesar de las dificultades de las mujeres para acceder a los cargos de poder y responsabilidad (Calvo y Rodríguez-Hoyos, 2012; Díez, Terrón y Anguita, 2006), desarrollan prácticas de liderazgo más distribuido, reforzando la participación de los diferentes agentes y, por tanto, dibujando el marco para la construcción de proyectos comunes (Hallinger, Dongyu y Wang, 2016).

Una tercera dimensión es la estructural, referente a los elementos formales de funcionamiento (González, 2009; López-Yáñez et al., 2002). Más concretamente, atiende a la distribución del trabajo, la configuración de unidades organizativas y los mecanismos formales para tomar decisiones y coordinar la acción. Atendiendo a la estructura formal, encontramos que las escuelas poseen un armazón similar por pertenecer a un mismo contexto normativo (San Fábian, 2011). La mayoría de escuelas españolas se organizan por etapas, ciclos y cursos, y poseen similares órganos de coordinación (equipos de ciclo, comisión de coordinación pedagógica) y gestión (equipo directivo, claustro y consejo escolar).

Los procesos internos de mejora requieren aumentar la autonomía de los centros educativos ${ }^{1}$ y potenciar la apertura de espacios de trabajo en los que los docentes puedan pensar en equipo y actuar juntos (Day, 2015; Kools y Stoll, 2016; Oria, 2009; Walker, Lee y Bryant, 2015). Se trata de definir un entramado organizativo que ponga a disposición de dichos proyectos recursos, espacios y tiempos (Bolívar, 2014; Gather y Maulini, 2010; San Fabián, 2011). De un lado, requiere repensar las estructuras formales existentes hacia fórmulas de responsabilidad compartida, devolviendo a algunos espacios formales su capacidad de influir, despojada por la LOMCE. De otro, precisa la creación de nuevos espacios de "colegialidad radical" (Fielding y Moss, 2011) que posibiliten relaciones horizontales entre los miembros de la comunidad educativa.

Complementariamente, apostamos por reformular los tiempos escolares, definidos actualmente por su carácter burocrático

\footnotetext{
Entendemos la autonomía desde la lógica pedagógica expuesta en el trabajo de Gairin (2008), sustentada en una gestión pública de la educación donde la autonomía constituye un elemento clave para que cada escuela construya su propio proyecto a partir de la cogestión de la educación por sus miembros y de la democratización en la toma de decisiones. Esta lógica se aleja de un enfoque neolibral pues reivindica una autonomía únicamente en el terreno pedagógico y organizativo que evite los mecanismos que generan competencia, insolidaridad y desigualdad económica y social.
} 
y por la idea de la hora como unidad de trabajo. Esta organización del tiempo, denominado "objetivo" (Bates, 2018), aparece como predecible, uniforme y limitado a secuencias cortas para responder a los criterios de productividad, control y eficiencia. En contraposición, es imprescindible pensar en un tiempo flexible que se define por lo vivido y por la experiencia educativa (Vázquez, 2007), donde la acción, el diálogo y los propios sujetos determinen su extensión.

Finalmente, cabría mencionar la dimensión de procesos y proyectos educativos. Una de las claves del cambio es la existencia de unas finalidades y procesos de planificación educativa compartidos, que otorgan dirección al proyecto y sostienen la acción educativa (Kools y Stoll, 2016; Pascual et al., 2016). Desde esta perspectiva comunitaria, los procesos de investigación-acción constituyen una estrategia clave de mejora (Colás-Bravo et al., 2014) pues permiten iniciar ciclos de reflexión y diálogo sobre las acciones y procesos de la escuela para finalmente construir una narrativa compartida.

\section{Metodología}

Este trabajo se inspira en la investigación etnográfica (Restrepo, 2016), dado que nuestro propósito es comprender en profundidad y "desde dentro" el funcionamiento y la progresiva transformación de las dimensiones de la organización escolar que han posibilitado desarrollar una innovación educativa que involucra a toda la escuela.

El centro objeto de estudio es el CEIP Manuel Llano, de titularidad pública y situado en una zona periférica de Santander que acoge a alumnado heterogéneo a nivel social, cultural y económico. Nuestra relación con esta escuela comienza en 2010, cuando iniciamos un proyecto de investigación sobre Voz del Alumnado ${ }^{2}$. Este comenzó como una experiencia de aula, implicando a dos grupos de $5^{\circ}$ de Primaria, para en los años subsiguientes ir involucrando a cada vez más alumnado y profesorado hasta convertirse en un proyecto de centro. En estos años, el equipo de investigación fue transfiriendo gradualmente la responsabilidad de las iniciativas de participación a los actores del centro, pero sin perder cierta continuidad de contacto. Tras ocho años, dos de las investigadoras retornamos al centro para realizar una última inmersión etnográfica con objeto de analizar uno de los proyectos innovadores en los que cristalizó el primer trabajo y que, en su singladura ya autónoma, la escuela desarrolla a nivel de centro desde el curso 2014-2015: las Tutorías Compartidas.

Esta iniciativa, encuadrada en el marco de la Acción Tutorial, se orienta a mejorar la convivencia en el centro. Quincenalmente se forman grupos de tipo inter-nivelar (alumnado de distintos cursos), que son acompañados por un docente distinto a su tutor, para realizar actividades en torno a temáticas definidas por el interés del alumnado y su relevancia social (el año del trabajo de campo se eligió la igualdad de género). Se persigue generar situaciones que contribuyan a desarrollar el sentido de pertenencia, la ayuda entre iguales y, especialmente, la creación de vínculos (PAT del CEIP Manuel Llano). Los profesionales que participaron en el proyecto de investigación de Voz del alumnado son los que han impulsado dentro del centro la iniciativa de las Tutorías Compartidas (equipo directivo y dos tutores que actualmente forman parte del equipo impulsor de Tutorías, del que hablaremos posteriormente en resultados). Este proyecto innovador de Tutorías Compartidas que ha sido objeto de estudio

\footnotetext{
2 Susinos, T. (Dir). Análisis de los procesos de inclusión/exclusión educativa en la educación obligatoria. Desarrollo de proyectos locales de cambio y mejora escolar. MICINN, Ref. EDU2008-06511-C02 02/EDUC.
}

sirve de marco para analizar algunas claves organizativas y culturales que han posibilitado la institucionalización de este tipo de iniciativas en el quehacer del colegio.

La primera fase del proceso de investigación etnográfica es la negociación y acceso al campo. Para este fin, antes de iniciar la investigación contactamos con la dirección del centro educativo, con la que ya manteníamos un contacto fluido, y explicamos de manera detallada el propósito de este proyecto y los requerimientos de participar. Una vez realizado este primer contacto, se desarrolló otra sesión informativa con los docentes implicados para explicar el proyecto y también con objeto de negociar los momentos de observación que ocurrirían durante todo el curso académico.

Respecto a la fase de trabajo de campo, una condición indefectible en etnografía refiere a la realización de una estancia prolongada en el campo y a la vinculación estrecha con las personas que son objeto de estudio (Velasco y Díaz de Rada, 2006). Así, realizamos el trabajo de campo a lo largo del curso 2017-2018 acudiendo de manera quincenal al centro educativo.

Las técnicas de recogida de información utilizadas corresponden a las propias de la etnografía: la observación participante, los grupos de discusión (GD) y el análisis documental.

Las observaciones de las actividades desplegadas se realizaron de manera continuada durante el curso, permitiendo recoger los datos en su medio natural durante un tiempo prolongado (Flick, 2018), así como mantener un contacto constante con los propios sujetos observados. En total se realizaron 8 observaciones, una por cada sesión de Tutoría Compartida realizada. En estas observaciones participaron las dos investigadoras, lo que permitió abarcar la observación de más grupos de Tutoría y, por ende, la construcción de dos diarios de campo por sesión. Los instrumentos utilizados fueron las notas de campo y los diarios (aproximadamente 10 páginas por cada observación). Asimismo, esta indagación etnográfica tiene una expresa vocación de explorar las percepciones de los actores implicados, y situarlas en el contexto en el que alcanzan significación (Cefaï, 2016). Para ello, concluido el curso escolar, realizamos 4 grupos de discusión (GD) de 6 participantes cada uno, que estuvieron conformados por profesorado (maestros, orientadora, jefa de estudios y directora) y por alumnado (desde $1^{\circ}$ de Primaria hasta $6^{\circ}$ ). Optamos por esta técnica dada su capacidad para ahondar en interpretaciones, a veces complementarias y otras confrontadas, acerca de las experiencias y por la posibilidad para capturar un número elevado de percepciones. Estos GD tuvieron un carácter semi-estructurado, de modo que utilizamos un guion previo con los tópicos a debatir que, no obstante, era lo suficientemente abierto como para permitir recoger algunos asuntos, inicialmente no previstos, que emergían durante el diálogo (Kamberelis, Dimitriadis y Welker, 2011). Estos grupos de discusión fueron grabados y posteriormente transcritos para facilitar el análisis.

Finalmente, se analizaron algunos documentos institucionales del centro (PEC y PAT) que suministraban contexto y suponían un apoyo a la observación.

La finalidad de la etnografía escolar va más allá de realizar una buena descripción de la cultura y dinámicas escolares y se orienta, prioritariamente, a la interpretación (Woods, 1987). Consecuentemente, realizamos un análisis de los datos a partir de un proceso de codificación temática, empleando estrategias de tipo inductivo y deductivo (Flick, 2018). Esto es, partimos de un esquema inicial de categorías a analizar, pero el trabajo desarrollado con los datos exigió redefinir algunas de esas categorías y códigos durante el proceso de análisis. Las categorías resultantes que articulan el análisis son las siguientes: 
Tabla 1.

Categorías de análisis.

\begin{tabular}{ll}
\hline Categorías & Descripción \\
\hline Cultura escolar & $\begin{array}{l}\text { Informaciones y observaciones acerca } \\
\text { de los supuestos, valores y concepcio- } \\
\text { nes pedagógicas que explícita o implí- } \\
\text { citamente articulan la organización y } \\
\text { acción del centro. }\end{array}$
\end{tabular}

Relaciones Declaraciones y observaciones sobre las relaciones formales e informales que determinan las redes de interacción y los flujos de comunicación.

Estructuras Informaciones y observaciones acerca de estructuras, tareas, roles y funciones que dan forma al entramado institucional.

Procesos y proyec- Reflexiones y observaciones sobre los tos

procesos de planificación y desarrollo de los procesos de enseñanza-aprendizaje.

Entorno

Informaciones y observaciones acerca de las relaciones con el entorno inmediato y mediato.

Fuente: elaboración propia.

\section{Resultados}

Este epígrafe se articula alrededor de cuatro apartados: cultura del centro alineada con un enfoque inclusivo; relaciones sustentadas en la colaboración, el intercambio y la apertura a la comunidad; estructura, tareas y roles al servicio del diálogo y la toma de decisiones compartida; y procesos y proyectos educativos que conectan con la cultura comunitaria del centro. Por motivos de espacio no abordamos todos los elementos constitutivos de cada una de las dimensiones, mas analizamos los aspectos nucleares que definen cada una de las dimensiones en el caso analizado.

\section{Cultura del centro alineada con un enfoque inclusivo}

La cultura escolar refiere a aspectos declarados, pero también a otros implícitos, no existiendo siempre correspondencia entre ambos planos (González, 2009). En el caso del Manuel Llano comprobamos cómo ambos se alinean, de manera que el ideario recogido en el PEC refleja las creencias, finalidades y prácticas encontradas en nuestro estudio. Así, una seña de identidad en este centro es su preocupación por que todos los niños se sientan parte de la escuela y, consecuencialmente, por buscar constantemente formas de atender a la diversidad (Slee, 2011):

Nuestro PEC se fundamenta en los principios de equidad, calidad y diversidad. Las metodologías que pretendemos implantar como forma generalizada de actuación favorecen la inclusión de todos los alumnos y la celebración de las diferencias (PEC Manuel Llano).

Es por lo que las innovaciones emprendidas se orientan a transformar el centro en un entorno acogedor que favorezca el acceso, la participación y el aprendizaje de todos los alumnos.

Generar en los alumnos un sentido de pertenencia a la escuela y también que tengan una participación alta (GD_ Orientadora).
Estos valores y prácticas identitarias se han entramado con el discurrir del tiempo, precisando espacios destinados al intercambio y sintonización de creencias y significados. Por tanto, estos rasgos hoy naturalizados, son fruto de un recorrido de muchos años de interrogarse a sí mismos sobre lo que se hace en el centro, sus fines y sus formas.

Así que esto es un trabajo de años. Yo entiendo que a la gente que lo ve la primera vez le abrume. Pero esto es un trabajo de años (GD_maestro).

Como comentan algunas maestras veteranas, es un colegio que no siempre se orientó por estas finalidades ni se caracterizó por su organización comunitaria (Fielding y Moss, 2011), existiendo una distancia entre lo declarado y algunas prácticas que se implementaban. Dos circunstancias fundamentales activaron el cambio: una nueva dirección (que abordaremos posteriormente) y la colaboración con el equipo de investigación de la Universidad de Cantabria. La participación en el proyecto de voz del alumnado supuso un punto de inflexión para el centro, que consolida su compromiso con los principios inclusivos y de la participación:

Surge como un proyecto de la Universidad para que aprendiéramos a utilizar oportunidades de participación del alumnado en clase... había un quinto con una relación muy mala y vimos la oportunidad de pensar en cómo podíamos utilizar esas oportunidades de participación para mejorarla ese año. Era todo por esa idea, en esa aula, fue fructífero y nos pareció muy interesante y el año siguiente se unieron los dos quintos (GD_Orientadora).

Con este empuje externo, el centro comienza un proceso de indagación que se prolonga hasta nuestros días y que tiene como propósito desarrollar progresivamente innovaciones educativas que caminan sobre estos principios inclusivos. De manera progresiva pero constante los distintos agentes del centro emprenden un camino que tiene el mismo destino. El fragmento siguiente ilustra cómo el alumnado asume esta filosofía de la inclusión:

Alumna: Somos una mezcla heterogénea.

Investigadora: ¿qué significa eso?

Alumna: Que hay una mezcla, que hay una diversidad.

Investigadora: Si le tenéis que contar a alguien qué son las tutorías compartidas, ¿qué le decís?

Alumno: Es una actividad de este cole para aprender todos en grupo y aportar todos.

Alumna: Es una actividad que sirve para estar todos participando y para apoyar a los demás.

(GD_alumnado)

Consecuentemente, los procesos reflexivos y de revisión continua de las prácticas escolares se integran en la dinámica del centro y las disonancias entre lo explícito y lo tácito se tornan cada vez más pequeñas:

La participación en tales proyectos, ha establecido dinámicas de reflexión continua sobre nuestra labor docente lo que, al mismo tiempo, ha originado el cuestionamiento de nuestro trabajo a través de dinámicas de evaluación continua de la práctica docente (PEC Manuel Llano). 
Nuestras observaciones durante la estancia en el centro nos llevan a pensar que existe una cultura compartida y, de algún modo, todos se sienten comprometidos con convertir el centro en una escuela para todos. No obstante, es cierto también que no todos los maestros comparten el mismo nivel de implicación, ni trasladan de la misma manera dichas formas de trabajo inclusivas a nivel de aula. En cualquier caso, los maestros más titubeantes y aquellos que llegan nuevos al centro reciben el acompañamiento de los "equipos impulsores", así como del equipo directivo, quien reconoce expresamente su intención de que, respetando el estilo de cada maestro, nadie se extravíe de esas finalidades inclusivas del centro.

Estos lineamientos definen culturalmente al centro y podemos ver de manera nítida cómo permean su funcionamiento de manera cotidiana.

\section{Relaciones sustentadas en la colaboración, el intercambio y la apertura a la comunidad}

La cultura participativa del Manuel Llano cristaliza en relaciones entre sus miembros apoyadas en la cooperación y en el sentido de comunidad (Fielding y Moss, 2011). Por ello, se plantean actividades y proyectos que implican el trabajo colaborativo de profesores, alumnos, familias y otros agentes del entorno.

Se busca permanentemente aumentar el nivel de participación de todos sus miembros: de los alumnos tanto en las aulas como en el centro, de los profesores a través del trabajo colaborativo y de las familias y el resto del entorno en su implicación en la escuela (PEC Manuel Llano).

En el caso de las Tutorías compartidas, por ejemplo, los alumnos participan activamente en cada una de las sesiones desarrolladas, valorando positivamente que se de importancia a su opinión (Mitra, 2018):

En la última tutoría hicimos unos post-it donde pusimos nuestra tutoría favorita y lo que queríamos hacer en la siguiente tutoría (GD_alumnado).

Es habitual en el funcionamiento cotidiano del centro que familias y otros agentes de la comunidad participen en las distintas iniciativas propuestas.

Maestra: el año pasado participaron mucho las familias en las Tutorías Compartidas.

Directora: no hay ningún rechazo, nadie viene a decir: oye, estáis perdiendo una hora de clase de matemáticas. Yo creo que también están tan acostumbrado que ya lo ven normal.

Orientadora: yo creo que se ve a veces que trasciende el espacio del cole. Hay una fiesta y vienen antiguos alumnos...

(GD_profesorado)

La escuela también se abre al entorno y colabora con personas y asociaciones para desarrollar las diferentes iniciativas. Este año en las Tutorías Compartidas participaron diferentes mujeres de la comunidad en una sesión destinada a fracturar los estereotipos de género asociados al desempeño profesional:

Vinieron unas chicas que tenían un trabajo que tradicionalmente hacían los hombres. Vino una señora que trabajaba en el parque de Cabárceno que daba de comer a los animales.
O una chica que era camionera, que eso es algo que siempre se ha visto que hacen los hombres (GD_alumnado).

Esta voluntad colaborativa se percibe ilustrativamente en la acogida que realizan a los nuevos profesores que reciben cada año, con la vocación de coadyuvar a que conozcan y asimilen la cultura del centro, se acomoden a la línea pedagógica y, en definitiva, puedan involucrarse en los distintos proyectos que el centro pone en marcha. De otro lado, se entiende que los nuevos docentes también pueden aportar planteamientos, experiencias e ideas al proyecto común.

A nadie se le deja solo. A un profesor que ha llegado nuevo, o un interino, o un definitivo que tampoco se siente muy cómodo... procuramos poner a dos profesores a trabajar juntos: uno un poco más experimentado y el otro. En cuanto ven las dinámicas entran al juego sin ninguna dificultad (GD_directora).

Esta cultura de la colaboración no es, sin embargo, fortuita, y precisa desplegar contextos facilitadores, así como espacios y tiempo para el intercambio, que permitan que los diferentes miembros vayan involucrándose en las dinámicas de trabajo escolar.

La base es intentar generar un buen ambiente también en el profesorado. Yo creo que sí dedicamos tiempo para trabajar estas cosas: en la sala de profesores siempre hay un ambiente cordial, siempre hay algo de comer, organizamos salidas... es un sitio muy vivo. Entonces yo creo que la clave para cualquier cosa curricular, antes de eso, es crear un ambiente. Y eso facilita todo, no es casual (GD_jefa de estudios).

El papel del equipo directivo, junto con la orientadora, ha resultado fundamental en la dinamización de estas iniciativas y procesos de cambio, y también en la generación de las estructurales espacio-temporales que posibilitan la coordinación y el diálogo. En nuestra estancia en el centro descubrimos una dirección que atesora un conocimiento grande del contexto, que establece una relación estrecha con todo el claustro, que sostiene planteamientos claros y consistentes, pero también flexibles y abiertos y que, en definitiva, ejerce un liderazgo distribuido que ha servido de impulso para la mejora (Diamond y Spillane, 2016).

\section{Estructura, tareas y roles al servicio del diálogo y la toma de decisiones compartida}

La escuela objeto de análisis posee las estructuras de coordinación comunes al resto de escuelas (González, 2009, San Fábian, 2011). Sin embargo, haciendo uso de su autonomía genera nuevos espacios al servicio de la cultura participativa: los equipos impulsores.

Tenemos creadas unas estructuras, los equipos impulsores. Nos parecía que la mejor manera es compartir, y compartir quiere decir que haya un grupo de profesores que trabaja sobre un tema, otro sobre otro, otro sobre otro...y todo eso se comparta. [...] lo que hacemos es que cada uno de los equipos impulsores esté formado también por gente de todos los ciclos y niveles, desde infantil hasta los que estamos en $5^{\circ}$ y $6^{\circ}$. [...] para cada uno de los equipos tenemos generados unos espacios y tiempos para que se reúnan (GD_jefa de estudios). 
En otras palabras, el proyecto educativo de este centro ha impulsado a sus miembros, liderados por el equipo directivo, a generar un espacio de trabajo caracterizado por la existencia de relaciones horizontales de toma de decisiones (Kools y Stoll, 2016), que excede el contexto-aula y que pone a disposición del proyecto recursos, espacios y tiempos (Gather y Maulini, 2010; San Fábian, 2011).

Yo creo que la clave para que este tipo de actividades salga es generar espacios y tiempos en el centro, generar espacios de participación entre el profesorado. Si no generas esos espacios te come el día a día. Necesitas un espacio para pensar y diseñar esas actividades. Tienes que buscar el espacio para compartir, si no compartes es imposible (GD_directora).

Es también reseñable, en este proceso, cómo el tiempo de los docentes (horas de docencia, tiempo de preparación, de permanencia, de sustituciones, de apoyo, etc.), es redefinido en aras de promover espacios de encuentro que tengan un valor pedagógico. No obstante, es un proceso que no ha estado exento de dificultades. A la alta movilidad docente, se añade la decisión de la Administración educativa en Cantabria de aumentar el número de horas de docencia en el aula, en detrimento de las horas de planificación y coordinación. Esta decisión externa influye en la organización escolar, empujando a las escuelas a inscribirse en una lógica eficientista y a los docentes a vincularse al aula desde un modelo técnico. En el Manuel Llano, sin embargo, a través de los grupos impulsores consiguen fracturar la tendencia de abandonar lo organizativo en beneficio del aula, sobreponiéndose a los óbices impuestos por la Administración y fortaleciendo la idea de comunidad.

En este sentido, la distribución horaria rompe con la lógica de la hora como unidad para dar paso a una planificación flexible, de carácter subjetivo (Vázquez, 2007).

Se coge toda la etapa de primaria y entonces ya se decide tener un espacio, para que pueda permitir ese trabajo. [...] Que todo el mundo tiene tutoría en esa hora. Entonces eso facilita muchísimo, esa organización facilita mucho, que todo el mundo puede incorporarse a esa actividad (GD_directora)

Esta reorganización del horario se ha encontrado con una barrera administrativa pues, aun reconociendo la relevancia de la acción tutorial en el marco normativo, las autoridades en Cantabria optaron por prescindir en el horario escolar de la hora adscrita a la tutoría. No obstante, el Manuel Llano reconquista su autonomía pedagógica para redefinir los aspectos de la organización en función de los proyectos que la escuela considera de valor para el desarrollo integral del alumnado.

Si miramos la normativa, la Consejería no da una hora en el horario para tutoría... pero sí que deja un par de horas, con cierta flexibilidad, que normalmente van en la carga curricular. Pero nosotros como centro hemos apostado una hora por ella. [...] Es una apuesta que organizativamente es compleja, lo cual quiere decir que realmente creemos en ellos sino no se haría. (GD_jefa de estudios).

Por otro lado, la edad deja de ser un clasificador del alumnado: los grupos-aula se reformulan para dar lugar a nuevas posibilidades de conocimiento y vínculo entre el alumnado de diferentes edades y para abrir las posibilidades de relación entre diferentes docentes y alumnado, donde el equipo docente al completo reconfigura sus roles y funciones habituales con el propósito de servir al proyecto común.

En esta clase están organizados en cuatro grupos de cuatro alumnos/as cada uno. Los grupos son mixtos en cuanto a edad y sexo (Observación II).

Y además añadiríamos también las relaciones que se crean con profesores que los niños no conocían de manera normal dentro de las clases. Claro, dentro de tu grupo de tutorías compartidas tienes niños desde primero hasta sexto. Entonces hay profesores que nunca han convivido con ese niño de primero que en ese momento sí que lo tienen (GD_maestro).

Como decíamos, más allá de las actividades puntuales, estos espacios generan una cultura escolar de encuentro, de convivencia y de reconocimiento. En conclusión, podemos señalar cómo los aspectos estructurales (roles, tareas, etc.) se han rediseñado en función de los proyectos comunitarios (Fielding y Moss, 2011).

\section{Procesos y proyectos educativos que conectan con la cultura comunitaria del centro}

La escuela se sirve de los procesos de planificación y proyectos para definir su identidad, sus características, necesidades y modos de acción. Este es un proceso edificado a lo largo del tiempo, resultante de las relaciones y acuerdos establecidos e influenciado por el entorno.

Yo creo que hay que remontarse al año 2011. [... [ La pirámide era así, primero un quinto. Al año siguiente se fusionaron los dos sextos. Después G. y yo lo hicimos extensible al ciclo. El siguiente año el tercer ciclo, $5^{\circ}$ y $6^{\circ}$, se unió. Luego ya al año siguiente se unió un cuarto, lo recuerdo perfectamente (GD_maestro).

En el anterior fragmento se aprecia el componente histórico de la experiencia de tutorías compartidas y cómo gracias al diálogo y la construcción colegiada el proyecto crece hasta convertirse en una seña de identidad. Es este acuerdo en las finalidades y modos de acción el que sostiene en el tiempo el proyecto educativo (Kools y Stoll, 2016). Sin embargo, los procesos de cambio son lentos y no existen atajos. Así, las tutorías compartidas han ido progresivamente ambicionando involucrar a más personas (profesores, alumnos, familias, entorno) y también gradualmente se han ido configurando las estructuras organizativas que las posibilitan.

Como decíamos, para el inicio y sostén de algunas acciones el acompañamiento por parte del equipo de investigación de la universidad fue fundamental. Esta oportunidad de pensar juntos evidenció el potencial formativo que tiene la creación de espacios compartidos de reflexión y facilitó a los maestros obtener una comprensión más profunda de los procesos de enseñanza-aprendizaje.

Posteriormente, se crearon en el centro los equipos impulsores. En el seno de estos equipos es donde se llevan a cabo los procesos de planificación educativa y, por tanto, las decisiones que adoptan definen el proyecto educativo común (Calvo y Rodríguez-Hoyos, 2012; González, 2009). Actualmente hay cinco equipos impulsores: competencia lingüística, competencia matemática, tutorías compartidas y convivencia, bilingüe y de salud. Todos ellos comparten el propósito de diseñar y planificar actuaciones inclusivas y ponerlas al servicio de todo el centro desarrollando procesos de reflexión para la indagación, mejora y transformación de los procesos educativos (Colás-Bravo et al., 2014). 
Investigadora: ¿cómo se deciden esos temas [de las tutorías compartidas]?

Maestro: el equipo impulsor. Admite sugerencias.

Orientadora: es verdad que este año con lo de la igualdad de género, era un tema que estaba muy a nivel de calle. Luego llegaron los ODS y vimos la posibilidad de juntarlo. Y el tema de los cuentos como que encajaba ahí... Eso son un poco las Primeras sesiones de trabajo del equipo impulsor.

Jefa de estudios: luego para cada uno de los equipos tenemos también generados unos espacios y tiempos para que se reúnan. Son los que se encargan de esa actividad (GD).

Vemos que este proceso de planificación y toma de decisiones requiere del imperativo dialógico (Fielding y Moss, 2011) para escuchar y tomar en consideración las formas de pensar divergentes. Además, en este proceso también son esenciales los procesos de reflexión durante y posteriormente a la acción. Para ello recurren a los espacios formales (Claustro) pero también a espacios subalternos en los que otros agentes, esencialmente el alumnado, participa del proceso de revisión y evaluación (Fielding y Moss, 2011; Mitra, 2018).

En esta experiencia encontramos un último momento que pudiera ser añadido al proceso reflexivo orientado a la mejora: compartir la experiencia, tanto dentro de los muros de las aulas como fuera, con la comunidad.

Maestro: procuramos siempre que cuando haya trabajos que tengan un producto final, ese producto final tenga visibilidad para el resto de la comunidad educativa [...].

Maestro: a mí me ha llegado información de amistades que no tienen nada que ver con el colegio que me preguntan: oye, que eso de las tutorías raras que hacéis en el colegio. Algo estaremos haciendo bien para que las familias comenten esta información fuera: en el colegio de mi hijo hacen esto. Así que es tanto para alumnado, para profesorado, para familias...

Jefa de estudios: yo iba a decir que trasciende un poco más... de fuera, otro centros, el CEP está interesado en el tema. Nos llaman para que vayamos a contarlo y tal. (GD)

En conclusión, se aprecia cómo el centro ha institucionalizado los procesos de reflexión continua en su tarea escolar, dotándose de herramientas para poder emprender de forma autónoma procesos de mejora.

\section{Conclusiones}

En este trabajo analizamos algunas claves de la organización escolar que facilitan el desarrollo de innovaciones educativas que involucran a todo el centro y que se ponen al servicio de los miembros de la comunidad educativa y sus proyectos.

Cabe señalar que nuestros hallazgos se alinean con los asuntos que están irrumpiendo con más fuerza en la investigación sobre organización escolar de los últimos años: liderazgo distribuido (Day, Gu y Sammons, 2016; Diamond y Spillane, 2016), autonomía pedagógica (Oria, 2009; Saloio y Morales, 2107; Serrano y Martín-Cuadrado, 2017), organización escolar democrática (Fielding y Moss, 2011; Segal et al., 2017), cultura profesional de la colaboración (Berg, 2018), etc.

En primer lugar, nuestra investigación parte del reconocimiento de que la escuela constituye una realidad cultural y política (Calvo y Rodríguez-Hoyos, 2012), resultando imprescindible en los procesos de innovación la revisión de los presupuestos y creencias que subyacen a lo que se hace en la escuela. Así, el
CEIP Manuel Llano despliega procesos de interrogación constante sobre sus políticas y prácticas que contribuyen a convertir el centro en un escenario abierto a las mejoras. En ocasiones, para desencadenar este proceso indagatorio se necesita algún empuje externo a la organización, como en este caso fue un equipo de investigación de la Universidad de Cantabria.

En segundo lugar, conviene señalar que estos procesos de re-culturación son consecuencia de un recorrido reposado en el que se necesitan condiciones para movilizar cambios y mejoras genuinas que calen hondamente en la cultura del centro. De esta suerte, la apertura de espacios de intercambio, reflexión y toma de decisiones compartida entre los diferentes profesionales aparece como otra clave irrenunciable en los procesos de mejora. Esta cultura de la colaboración precisa, a su vez, algunas condiciones materiales y estructurales (clima, recursos, tiempos, espacios) que posibiliten la indagación compartida, cuestión cada vez más dificultosa como consecuencia de la última ley educativa que devuelve a un modelo profesional técnico. El equipo directivo del Manuel Llano supera estas rémoras creando nuevos espacios al servicio de la cultura colaborativa: los equipos impulsores y las horas comunes para actividades a nivel de centro. La idea llave de los equipos impulsores radica en que exista un colectivo de profesores, no muy numeroso, capaz de tomar la iniciativa en un ámbito de innovación y producir un efecto de contagio en el resto de docentes. Para ello se generan estructuras físicas en el horario lectivo que procuran al profesorado espacios de encuentro destinados a realizar esta labor de coordinación, reflexión conjunta (Kools y Stoll, 2016). Por otro lado, también consiguen flexibilizar los tiempos escolares (Vázquez, 2007) y estipular un momento semanal, en la que todo el centro comparte en su horario una hora de tutoría.

Igualmente, comprobamos el papel que desempeña la dirección escolar en la dinamización de estos procesos internos de mejora y en la reconquista de los procesos de autonomía institucional (Saloio y Morales, 2017). Si bien las mujeres miembros de la dirección animan estas iniciativas y generan las estructuras que las posibilitan, descubrimos que el liderazgo se comparte con otros miembros de la institución, entendiéndolo como un producto de actividades que desarrollan conjuntamente (Diamond y Spillane, 2016). Constatamos, de otra parte, los hallazgos de otras investigaciones que relacionan este liderazgo distribuido con el uso que realizan las mujeres del poder y la autoridad hacia formas más empáticas y cooperativas (Díez et al., 2006; Hallinger et al., 2016).

Huelga destacar, finalmente, la interdependencia de las diferentes dimensiones analizadas y cómo el movimiento de una de las llaves activa el engranaje que da lugar a que el total de la organización comience a caminar hacia la mejora.

\section{Referencias bibliográficas}

Álvarez-Arregui, E. y Arreguit, X. (2019). El futuro de la Universidad y la Universidad del Futuro. Ecosistemas de formación continua para una sociedad de aprendizaje y enseñanza sostenible y responsable. Aula Abierta, 48, 4, 447-479

Antúnez, S., y Gairin, J. (2013). La organización escolar: práctica y fundamentos. Barcelona: Graó.

Bates, A. (2018). Readiness for School, Time and Ethics in Educational Practice. Studies in Philosophy and Education, 38, 1-16.

Berg, J. (2018). Leading together/syncing behavior strategies. Educational Leadership, 76(1), 74-85.

Gairin, J. (2008). La autonomía de centros educativos en España: entre declaraciones discursivas y prácticas sobrerreguladas. En García-Albaladejo (coord.), La autonomía de los centros escolares (pp. 81-130). Madrid: Ministerio de Educación Cultura y Deporte. 
Bolívar, A. (2014). Building school capacity: Shared leadership and professional learning communities. A research proposal. International Journal of Educational Leadership and Management, 2(2), 147-175.

Bolívar, A., y Murillo, F. (2017). La escuela importa. Los efectos diferenciales de la escuela y el liderazgo en la equidad. En J. Weinstein y G. Muñoz (Eds.), Mejoramiento y liderazgo en la escuela. Once miradas (pp. 71-112). Santiago de Chile: CEDLES.

Calvo. A., y Rodríguez-Hoyos, C. (2012). Aportaciones de los estudios de las mujeres y del género a la organización escolar. El estado de la cuestión en España. Educación XX1, 15(1), 4360.

Cantón, I., y Pino, M. (2014). Organización de Centros Educativos en la Sociedad del Conocimiento. Madrid: Alianza editorial

Cefaï, D. (2016). ¿Qué es la etnografía? Debates contemporáneos Primera parte. Arraigamientos, operaciones y experiencias del trabajo de campo. Persona y sociedad, 27(1), 101-119.

Colás-Bravo, P., Jiménez, J., y Rosado, J. (2014). La acción tutorial en la educación actual. Madrid: Síntesis.

Diamond, J., y Spillane, J. (2016). School leadership and management from a distributed perspective: A 2016 retrospective and prospective. Management in Education, 30(4), 147-154.

Day, C. (2015). International successful school principals project (ISSPP): Multi-perspective research on school principals. Nottingham: University of Nottingham.

Day, C., Gu, Q., y Sammons, P. (2016). The impact of leadership on student outcomes: How successful school leaders use transformational and instructional strategies to make a difference. Educational Administration Quarterly, 52(2), 221-258.

Díez, E., Terrón, E., y Anguita, R. (2006). La cultura de género en las organizaciones escolares. Motivaciones y obstáculos para el acceso de las mujeres a los puestos de dirección. Barcelona: Octaedro.

Fielding, M., y Moss, P. (2011). Radical education and the common school: A democratic alternative. United Kingdom: Routledge.

Flick, U. (2018). Designing qualitative research. United Kingdom: Sage.

Gather, M., y Maulini, O. (2010). La organización del trabajo escolar. Una oportunidad para repensar la escuela. Barcelona: Graó.

Gimeno, J. (2006). La reforma necesaria: Entre la politica educativa y la práctica escolar. Madrid: Morata

González, M. T. (2009). Organización y gestión de centros escolares: dimensiones y procesos. Madrid: Pearson Educación.

Grootenboer, P., Edwards-Groves, C., y Rönnerman, K. (2015). Leading practice development: Voices from the middle. Professional Development in Education, 41(3), 79-93.

Hallinger, P., Dongyu, L., y Wang, W. (2016). Gender differences in instructional leadership: A meta-analytic review of studies using the principal instructional management rating scale. Educational Administration Quarterly, 52(4), 567-601.

Hopkins, D. (2013). Exploding the myths of school reform. Victoria: Centre for Strategic Education.

Kamberelis, G., Dimitriadis, G. y Welker, A. (2017). Focus Group Research and/in Figured Worlds. En N. Denzin e Y. Lincoln (Eds.), The SAGE handbook of qualitative research (pp. 692-716). Thousand Oaks, CA: Sage Publications.

Kools, M., y Stoll., L. (2016), What makes a school a learning organisation?. Paris: OECD Publishing. http://dx.doi.org/10.1787/ 5jlwm62b3bvh-en.

Leithwood, K., Harris, A., y Hopkins, D. (2008). Seven strong claims about successful school leadership. School Leadership and Management, 28(1), 27-42.

Leiva, M., Gairin, J. y Guerra S. (2019). Prácticas de liderazgo de los directores noveles para la inclusión educativa. Aula Abierta, 48(3), 291-300.
López-Yáñez, J., Sánchez-Moreno, S., y Nicastro, S. (2002). Análisis de organizaciones educativas a través de casos. Madrid: Síntesis.

Martínez-Valdivia, E., García-Martínez, I., e Higueras-Rodríguez, M. (2018). El liderazgo para la mejora escolar y la justicia social. Un estudio de caso sobre un centro de educación secundaria obligatoria. Revista Iberoamericana sobre Calidad, Eficacia y Cambio en Educación, 16(1) 35-51.

Mitra, D. (2018). Student voice in secondary schools: the possibility for deeper change. Journal of Educational Administration, $56(5), 473-487$.

Mulford, B. (2006). Leadership and quality secondary education. Profesorado. Revista de currículum y formación del profesorado, 10(1), 1-20.

Oria, M. (2009). ¿Es posible la autonomía escolar? El papel de la dirección y de los proyectos del centro en los sistemas educativos francés, italiano y español. Educación XX1, 12(1), 151180.

Pascual, J., Larraguibel, D., Zenteno, D., y Guarda, F. (2016). Liderazgo escolar en tiempos de crisis. El caso de dos liceos del centro sur de Chile después del 27F. REICE. Revista Iberoamericana sobre Calidad, Eficacia y Cambio en Educación, 14(2), 45-62.

Peréz-Gomar, G. (2016). La matriz insonora del cambio educativo: la organización escolar. Perspectivas para su investigación. REXE-Revista de Estudios y Experiencias en Educación, 7(13), 43-60.

Porter. L., Lawler, E., y Hackman. R. (1975). Behavior in organizations. New York: McGrawHill.

Restrepo, E. (2016). Etnografía: alcances, técnicas y éticas. Bogotá: Envión Editores.

Saloio, M., y Morales, J. (2017). Class councils: Organization, leadership \& autonomy. Revista Lusofona de Educacao, 35(35), 101-118.

San Fabián, J. (2011). El papel de la organización escolar en el cambio educativo: la inercia de lo establecido. Revista de Educación, 356, 41-60.

Segal, A., Pollak, I., y Lefstein, A. (2017). Democracy, voice and dialogic pedagogy: The struggle to be heard and heeded. Language and Education, 31(1), 6-25.

Serrano, R., y Martín-Cuadrado, A. (2017). Diagnosis de la problemática actual de las direcciones escolares. Revista Complutense de Educacion, 28(4), 1193-1210.

Slee, R. (2011). The irregular school: Exclusion, schooling and inclusive education. United Kingdom: Taylor \& Francis.

Thomson, P., y Hall, C. (2015). 'Everyone can imagine their own Gellert': the democratic artist and 'inclusion'in primary and nursery classrooms. Education, 43(4), 420-432.

Vázquez, R. (2007). Reflexiones sobre el tiempo escolar. Revista Iberoamericana de Educación, 42(6), 1-11.

Velasco, H., y Díaz de Rada, Á. (2006). La lógica de la investigación etnográfica. Un modelo de trabajo para etnógrafos de escuela. Madrid: Trotta.

Walker, A., Lee, M., y Bryant, D. (2015). How much of a difference do principals make? An analysis of between schools variation in academic achievement in Hong Kong public secondary schools. School Effectiveness and School Improvement, 25, 602-628.

Woods, P. (1987). La escuela por dentro. La etnografía en la investigación educativa. Barcelona: Paidós. 